\title{
Hongos en semillas de Pinus montezumae Lamb. y Pinus greggii Engelm. ex Parl. almacenadas bajo dos humedades relativas
}

\section{Seed fungi of Pinus montezumae Lamb. and Pinus greggii Engelm. ex Parl. stored under two relative humidities}

\author{
Merari Sujey Vázquez López ${ }^{1}$, Mario Ernesto Vázquez Badillo1*, Adriana Antonio Bautista²
} y Arturo Mancera Rico ${ }^{1}$

\begin{abstract}
Fungi develop upon pine seeds during storage, and deteriorate their quality. The objective of this study was to identify the fungal genera that are associated with the seeds of Pinus montezumae and $P$. greggii in two relative humidities (HR). They were stored at 60 and $80 \% \mathrm{RH}$ at $5{ }^{\circ} \mathrm{C}$. The samplings were made for 180 days. Seed moisture content (HS), fungal free seeds (SLH) were assessed and fungal genera (GH) were identified by colony morphology. In HS and SLH, a completely randomized experimental design was applied and a comparison of means was made. All sources of variation were significant. The mean HS per species was 9.59 and $12.37 \%$ and SLH of 64.52 and $69.28 \%$ for $P$. greggii and $P$. montezumae, respectively. In the $P$. greggii seed stored at $60 \mathrm{HR}$, HS was $7.97 \%$, with $66.19 \%$ in SLH and at $80 \mathrm{HR}, 11.21 \%$ and $62.85 \%$ in SLH. For P. montezumae at $60 \mathrm{HR}$, the HS was $10.21 \%$ and $71.42 \%$ in SLH; at $80 \mathrm{HR}$, HS was $14.53 \%$ and $67.14 \%$ in SLH. The GH identified promote the deterioration of the seeds; they were: Alternaria sp, in P. greggii at $60 \mathrm{HR}$ it was not observed, Penicillium sp. increased and predominated after 120 days, Fusarium sp. was constant; Aspergillus sp. and Rizhopus sp., appeared sporadically
\end{abstract}

Key words: Storage, quality, fungi, relative humidity, Pinus spp., seed.

\section{Resumen}

Las semillas de pinos durante su almacenamiento pueden presentar hongos que deterioran su calidad. El objetivo de la presente investigación consistió en identificar los géneros fúngicos que se asocian a las semillas de Pinus montezumae y P. greggii en dos humedades relativas (HR). Se les almacenó a 60 y $80 \%$ de HR a $5{ }^{\circ} \mathrm{C}$. Los muestreos se hicieron durante 180 días. Se evaluó el contenido de humedad de semilla (HS), las semillas libres de hongos (SLH); y se identificaron los géneros de los hongos (GH) por la morfología de sus colonias. En HS y SLH se aplicó un diseño experimental completamente al azar y se hizo una comparación de medias. Todas las fuentes de variación fueron significativas. La HS media por especie fue de 9.59 y de $12.37 \%$; la SLH de 64.52 y $69.28 \%$ para $P$. greggii y $P$. montezumae, respectivamente. En la semilla de $P$. greggii almacenada a $60 \mathrm{HR}$, la HS fue de $7.97 \%$, con $66.19 \%$ en SLH y a $80 \mathrm{HR}$, de $11.21 \%$ y $62.85 \%$ en SLH. Para P. montezumae a $60 \mathrm{HR}$, la HS fue de $10.21 \%$ y de $71.42 \%$ en $\mathrm{SLH}$; a $80 \mathrm{HR}$, la HS fue de $14.53 \%$ y $67.14 \%$ en SLH. Los GH identificados que promueven el deterioro de las semillas fueron: Alternaria sp, que en $P$. greggii a $60 \mathrm{HR}$ no se observó; Penicillium sp incrementó y predominó a partir de los 120 días; Fusarium sp. fue constante; de forma esporádica se presentaron Aspergillus sp y Rizhopus sp.

Palabras clave: Almacenamiento, calidad, hongos, humedad relativa, Pinus spp., semilla.

Fecha de recepción/Reception date: 19 de noviembre de 2020

Fecha de aceptación/Acceptance date: 5 de mayo de 2021

1 Universidad Autónoma Agraria Antonio Narro. Centro de Capacitación y Desarrollo en Tecnología de Semillas. México.

${ }^{2}$ Universidad Autónoma Agraria Antonio Narro. Departamento de Fitomejoramiento. México.

*Autor para correspondencia; correo-e: marioe.vazquez@hotmail.com 


\section{Introducción}

La restauración de bosques degradados y tierras agrícolas se ha convertido en un aspecto principal de la conservación global (Christin et al., 2016). Varios países desarrollan métodos de preservación de semillas de especies forestales con la finalidad de mejorar su adaptación, crecimiento y calidad (Skrøppa y Fjellstad, 2017). Pinus montezumae Lamb. y Pinus greggii Engelm. ex Parl. son especies prioritarias de los bosques de coníferas en México pues revisten gran importancia económica, ecológica y social (Conafor-FAO, 2011). Además, la segunda es endémica de Norte América y está catalogada en estatus de vulnerabilidad según la International Union for the Conservation of Nature (Conabio, 2018).

Para la familia Pinaceae, la semilla es el principal recurso de propagación. Sin embargo, durante su almacenamiento diversos factores causan su deterioro: su contenido de humedad, la humedad relativa y la temperatura del ambiente (Wang y Beardmore, 2004). Otros agentes que favorecen el daño a dichas estructuras son los organismos vivos (Arguedas, 1997) como: hongos, bacterias, virus, nematodos y artrópodos; de ellos, los hongos son el grupo de patógenos de mayor transmisión, se desarrollan en la superficie, en su interior o en ambas partes y se propagan por medio de diferentes formas, como esporas y esclerocios (Neegaard, 1977).

La información sobre los patógenos asociados a las semillas forestales almacenadas es escasa, y los hongos resultan de interés porque los daños que ocasionan repercuten en su posterior establecimiento. Con base en lo anterior, se plantearon como objetivos identificar y cuantificar los géneros de hongos asociados a las semillas almacenadas de Pinus montezumae y Pinus greggii bajo dos condiciones de humedad relativa. 


\section{Materiales y Métodos}

La investigación se realizó en el Centro de Capacitación y Desarrollo en Tecnología de Semillas de la Universidad Autónoma Agraria Antonio Narro, en Saltillo, Coahuila, en junio de 2018. Las semillas de pino prieto (Pinus greggii) y pino real (Pinus montezumae) recolectadas en los municipios de Metepec y Calimaya respectivamente, en el Estado de México fueron proporcionadas por la Comisión Nacional Forestal (Conafor).

Se tomó una muestra representativa de un kilogramo en cada lote; por especie se utilizaron cuatro repeticiones de 50 semillas por humedad relativa (60 y $80 \%$ ) y para cada muestreo, los que se llevaron a cabo a los 0, 30, 60, 90, 120, 150 y 180 días. Las semillas se colocaron en bolsa de malla y se suspendieron sobre una tarima dentro de recipientes de plástico, para que durante el tiempo de almacenamiento las semillas no tocaran directamente las soluciones. Las humedades relativas (HR) se obtuvieron con sales según Winston y Bates (1960); para $60 \%$ de HR, se colocó $1.5 \mathrm{~kg}$ de sal de grano para los muestreos dos (30 días) y tres (60 días); mientras que, para los muestreos del cuatro al siete (90 a 180 días) se usaron $2.0 \mathrm{~kg}$. Para $80 \% \mathrm{HR}$, se agregó $0.5 \mathrm{~kg}$ de sal más $1 \mathrm{~L}$ de agua para los primeros dos muestreos y $1.1 \mathrm{~kg}$ más $2 \mathrm{~L}$ de agua para los muestreos del cuatro al siete.

El material así tratado, se almacenó en una cámara de enfriamiento marca Torrey modelo CV-32 a temperatura constante de $5{ }^{\circ} \mathrm{C}$. Las variables evaluadas fueron: contenido de humedad de la semilla (HS), que se calculó con base en ISTA (2004) con 15 semillas en promedio por cada repetición; las semillas se pusieron en cajas de aluminio con tapa, previamente pesadas; enseguida se pesaron las cajas con la semilla húmeda y se colocaron dentro de una estufa de secado SHEL-LAB modelo FX14-2, a $103^{\circ} \mathrm{C}$ por $16 \pm 1$ horas; posteriormente, se pesaron las cajas después del secado. Para todas las mediciones se utilizó una balanza analítica marca OHAUS modelo AV264. La HS se calculó por diferencia de peso y se expresó en porcentaje. 
La identificación y cuantificación de géneros de hongos $(\mathrm{GH})$ y semillas libres de hongos (SLH), se realizó mediante la técnica de crecimiento en medio de cultivo Malta Sal Agar (Moreno, 1988). Se utilizaron 10 semillas con cuatro repeticiones por muestreo, mismas que se desinfestaron con $\mathrm{NaClO}$ al $0.5 \%$ para $P$. greggii y al $1.0 \%$ para $P$. montezumae, durante 1 minuto para ambas especies; las diferencias en la concentración de $\mathrm{NaClO}$ se debe a que el tegumento de $P$. greggii es más delgado y sensible al hipoclorito de sodio, con respecto a $P$. montezumae. A continuación se hizo la siembra dentro de una campana de flujo laminar marca ALDER, con 10 semillas por caja Petri; posteriormente, se les depositó en una cámara de incubación marca SHEL-LAB modelo FX14-2 a temperatura de $28-30{ }^{\circ} \mathrm{C}$, por 7 días. Los $\mathrm{GH}$ se identificaron por la morfología de sus colonias con las claves taxonómicas de Barnett y Hunter (1998) y Moreno (1988). Para SLH se cuantificaron las semillas que no desarrollaron micelio y se registraron en porcentaje. La incidencia (I) de GH para los dos taxones de pino, se calculó mediante la siguiente fórmula (Abdullah y Al-Mosawi, 2010):

$$
I \%=\frac{\text { Cantidad de semillas en las que apareció el hongo }}{\text { Número total de semillas }} * 100
$$

Se aplicó un diseño experimental completamente al azar para humedad de semilla y semillas libres; se llevó a cabo una comparación de medias de Tukey $(P<0.05)$, con el paquete estadístico SAS (2002).

\section{Resultados}

El análisis de varianza mostró que para las fuentes de variación analizadas: especie, humedad relativa y tiempo hubo significancia de 0.01 . Para HS por especie, $P$. montezumae registró una media de $12.37 \%$, lo que fue estadísticamente diferente a P. greggii con $9.59 \%$; en SLH, P. montezumae obtuvo los mayores valores, con 69.28 $\%$, en comparación con $P$. greggii $(64.52 \%)$. La menor HS se determinó bajo la 
condición de $60 \mathrm{HR}$, P. montezumae presentó $10.21 \%$ y P. greggii $7.97 \%$; para 80 $H R$, se incrementó para la primera especie (14.53\%) y para la segunda, $11.21 \%$ de humedad de la semilla. En cuanto las semillas libres de hongos, en la condición de 60 HR $P$. montezumae registró $71.42 \%$ y $P$. greggii $62.85 \%$ y para el ambiente de 80 HR, el primer taxón, 67.14 \% y el segundo, $66.19 \%$.

Durante el tiempo de almacenamiento, la HS fue similar para ambas especies. En $P$. montezumae bajo la condición de humedad relativa al $60 \%$, se observó la menor HS a los 0 días (8.72\%); al 80 HR la mayor HS fue a los 180 días (16.42\%). Con respecto a SLH, el porcentaje más alto se identificó a los 30 días en condiciones de $60 \mathrm{HR}$ y, en $80 \mathrm{HR}$, a los 60 y 90 días (76.66 y $73.33 \%$ ). El comportamiento de $P$. greggii fue similar, ya que a los cero días bajo 60 HR se obtuvo el valor de HS más bajo (7.58 \%); para SLH fue a los 120 días a 80 HR con el valor más alto (75.6 \%), mientras que a $60 \mathrm{HR}$ los valores más destacados se observaron a los 150 y 180 días con 73.72 \%. Para ambas especies, la mayor HS se alcanzó a los 180 días en las dos condiciones de humedad relativa: P. greggii 9.08 y $P$. montezumae $13.06 \%$; finalmente, para SLH no hubo diferencias significativas entre las dos condiciones de humedad relativa (Figura 1).

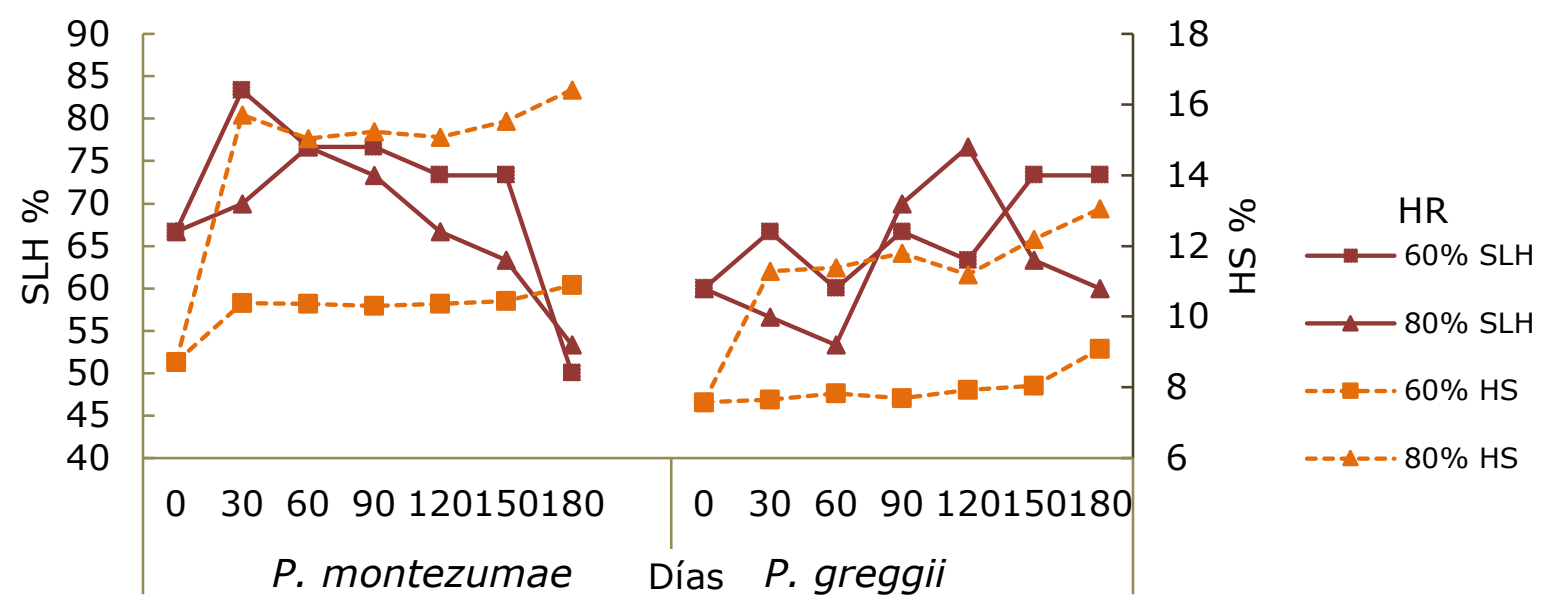

Figura 1. Respuesta de Pinus montezumae Lamb. y Pinus greggii Engelm. ex Parl., la humedad de la semilla (HS) y semillas libres de hongos (SLH) durante 180 días de almacenamiento a 60 y $80 \%$ de humedad relativa (HR). 
Los géneros de hongos presentes en ambas especies y humedades durante el almacenamiento fueron Fusarium sp., Penicillium sp., Alternaria sp., Rizhopus sp. y Aspergillus sp.; sin embargo, este último no se manifestó en $P$. greggii al $60 \%$ de HR. Al inicio del almacenamiento, la cantidad y variabilidad de géneros fue mayor, pero, al finalizar los muestreos Penicillium sp. fue predominante.

En P. montezumae al $60 \%$ de HR, Penicillium sp. presentó incidencias de $30 \%$ a los 180 días; y la máxima de Fusarium sp. fue a los 90 días (16.7 \%). Alternaria sp. y Aspergillus sp. disminuyeron hasta $0 \%$, en cambio Rizhopus sp. se mantuvo hasta los 180 días (Figura 2a). Al $80 \%$ de HR, Penicillium sp. dominó con una incidencia de 8.33 a $43.3 \%$, en cambio Fusarium sp. se verificó hasta el último muestreo; mientras que Aspergillus sp. al día 0 se mostró con 10 \% y Rizhopus sp con $2.33 \%$ (Figura 2). 


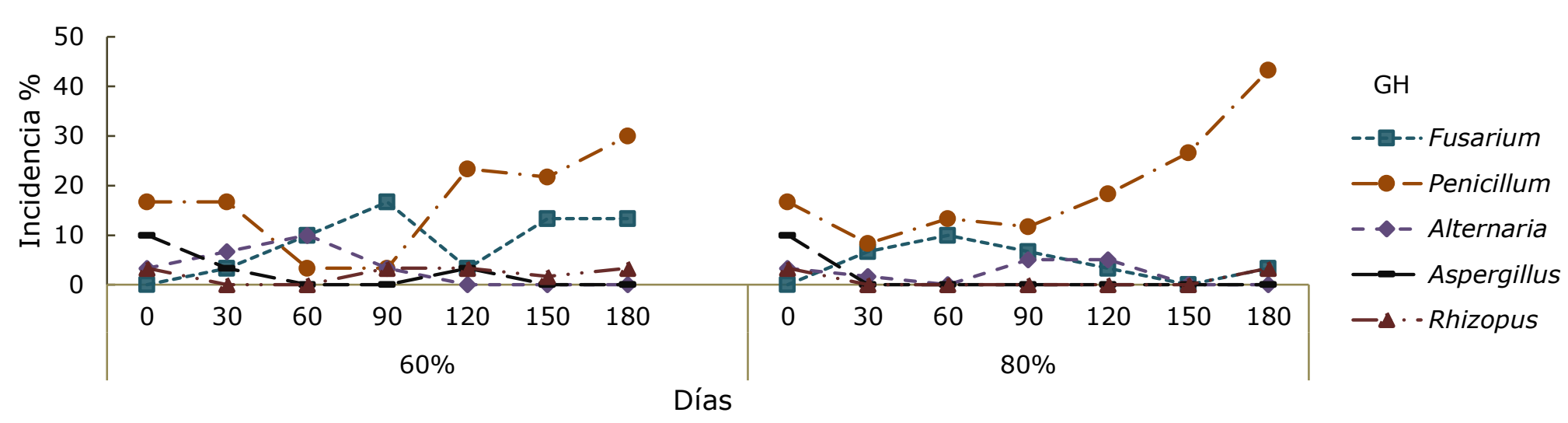

Figura 2. Porcentaje de incidencia de diferentes géneros de hongos (GH) en semillas de Pinus montezumae Lamb. durante 180 días de almacenamiento a 60 y $80 \%$ de humedad relativa. 
La incidencia de hongos en $P$. greggii fue similar a la de $P$. montezumae, a los 0 días Alternaria sp. dominó con 27 \% (Figura 3), pero disminuyó con el paso del tiempo. Fusarium sp. estuvo presente durante todo el almacenamiento. A la humedad relativa de $60 \%$, su máxima incidencia fue a los 60 días (23.3\%) y a la HR de $80 \%$ a los 30 días fue de $16.7 \%$; Rizhopus sp. solo se presentó al inicio del experimento En el ambiente de 60 \% de HR no se detectó Aspergillus sp., y la incidencia de Penicillium sp., fue mayor a 120 días con $33 \%$. En el ambiente de $80 \%$ de HR el mayor incremento de Penicillium sp. fue de $30 \%$ a 60 y 180 días, mientras que Aspergillus sp. solo se observó a los 150 días con $3.33 \%$. 


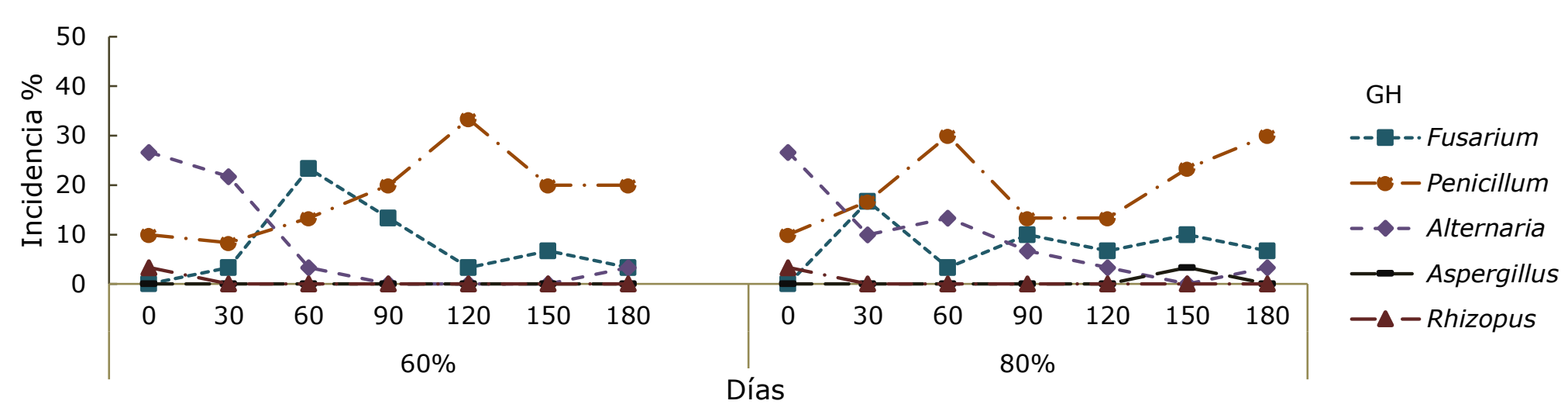

Figura 3. Porcentaje de incidencia de diferentes géneros de hongos (GH) en semillas de Pinus greggii Engelm. ex Parl. durante 180 días de almacenamiento a humedad relativa del 60 y $80 \%$. 


\section{Discusión}

La HS está en función directa de la humedad relativa y la temperatura del ambiente. Al tener la capacidad de perder o ganar humedad de acuerdo a las condiciones ambientales (Delouche, 1972), P. montezuma y $P$. greggii alcanzaron la humedad de equilibrio a los 30 días y valores máximos de 16.42 y $13.06 \%$, respectivamente; sin embargo, Conafor $(2017 a, b)$ recomienda que la humedad más apropiada para el almacenamiento en $P$. montezumae es de $8-10 \%$ y para $P$. greggii del 6-7 \%. La HS durante el almacenamiento de estas especies resultó ser un factor determinante para mantener la calidad de la semillas (SNICS, 2018). Estos porcentajes de humedad de la semilla favorecen la presencia de hongos y con ello, el proceso de deterioro por factores bióticos (Engels y Visser, 2007).

En referencia a la HR, Ortiz-Catón et al. (2011) establecieron que la actividad biológica de diversas cepas de hongos se incrementa cuando están en un intervalo de 81 - 92 \%. En Brassica sp., Suma et al. (2013) concluyeron que la viabilidad de las semillas y los parámetros de vigor de las plántulas se redujeron cuando se les sometió a una HR alta (75\%), lo que coinciden con los resultados obtenidos en el presente estudio, en el que se observó que a $80 \% \mathrm{HR}$ la incidencia de hongos fue mayor que a $60 \% \mathrm{HR}$, en las dos especies de interés.

Ceballos-Freire y López-Ríos (2007) señalaron que en Alnus acuminata Kunth (aliso), Guarea guidonia (L.) Sleumer (cedrillo), Juglans neotropica Diels (cedro negro), Retrophyllum rospigliosii (Pilg.) C. N. Page (chaquiro) y Cordia gerascanthus L. (solera) la alta humedad relativa (90 \%) provocó un aumento de la humedad de la semilla almacenada y, como consecuencia, se presentó un ataque severo de hongos de los géneros Penicillium y Aspergillus, los cuales afectaron 50 y $70 \%$ del material en conservación, respectivamente.

La tendencia de SLH para $P$. montezuma disminuyó drásticamente en las dos HR a partir de los 90 días; en $P$. greggii al 80 \%, la tendencia bajó a los 120 días, lo que coincide con investigaciones sobre semillas de Pinus spp. que indican la pérdida de 
viabilidad por el aumento de daños fisiológicos (Hilli et al., 2003; Du Hyun y Sim, 2018) durante el almacenamiento a largo plazo.

Los géneros determinados en ambas especies de pino sometidas a ambientes de 60 y $80 \%$ de HR durante los 180 días coinciden con la información aportada por Guerra et al. (2004) y Campo-Aranda et al. (2014) sobre la micobiota en semillas de Pinus spp. De acuerdo con Moreno (1988), los hongos de campo como Alternaria sp. y Fusarium sp., se desarrollan en humedades entre 90 y $100 \%$. De esta forma, Alternaria sp. estuvo presente en las semillas al inicio de los muestreos y disminuyó su incidencia conforme trascurrió el tiempo. Fusarium sp. se comportó de igual forma, fue mayor a $60 \%$ de $\mathrm{HR}$ que a $80 \%$ enlos dos taxones de pino evaluados. Al desarrollarse este género en la semilla, ocasiona que la calidad de la planta sea inferior, ya que daña al embrión antes de germinar y causa necrosis del hipocótilo y los cotiledones (Peterson, 2008; Solano-Bonilla y Brenes-Chacón, 2012).

En este estudio se presentaron hongos del género Penicillium sp., Aspergillus sp. y Rhizopus sp., catalogados como hongos de almacén con crecimiento en un intervalo entre 65 y $90 \%$ de HR. Penicillium sp. incrementó su incidencia en todos los ambientes y en ambas especies a partir de los 120 días, además Aspergillus sp. se identificó con mayor frecuencia en $P$. montezumae, pero en $P$. greggii solo se le identificó al $80 \%$ de HR. Rhizopus sp. se observó de forma esporádica en $P$. montezumae. Estos géneros disminuyen la calidad fitosanitaria de las plántulas, ya que causan pudriciones, reducción de crecimiento y muerte de plántulas (Borges y Urdaneta, 2010; Arguedas, 2011; Lee, 2011).

Gran parte de estos géneros de hongos son considerados saprofitos, algunos no siempre provocan daños directos en la semilla, pero se reconoce que cuando la incidencia es muy alta, el vigor y la viabilidad de las semillas tienden a disminuir (Mittal et al., 1990). 


\section{Conclusiones}

Las condiciones de almacenamiento de semillas de $P$. montezuma y $P$. greggii con alta humedad relativa aumentan la incidencia fúngica de géneros como: Fusarium sp., Aspergillus sp., Alternaria sp., Rhizopus sp. y Penicillium sp., los cuales se han identificado como causantes de deterioro.

Los ambientes fríos $\left(5^{\circ} \mathrm{C}\right)$ y secos $(60 \% \mathrm{HR})$ pueden ser seguros para el almacenamiento de semillas de $P$. montezumae y $P$. greggii, ya que son las condiciones en las que menor porcentaje de humedad de la semilla se registra y en consecuencia hay menos incidencia de hongos causantes del deterioro.

\section{Agradecimientos}

A la Universidad Autónoma Agraria Antonio Narro (UAAAN) por el apoyo con equipos y laboratorios durante el almacenamiento de las semillas. Al Consejo Nacional de Ciencia y Tecnología (Conacyt) por el apoyo económico para la realización de la investigación, y a la Comisión Nacional Forestal (Conafor) por la aportación de semilla.

\section{Conflicto de intereses}

Los autores declaran no tener conflicto de intereses.

\section{Contribución por autor}

Merari Sujey Vázquez López: establecimiento del proyecto, toma de datos, identificación de géneros, análisis de la información estructuración y redacción del manuscrito; Mario Ernesto Vázquez Badillo: estructuración del proyecto, planeación, selección de variables y revisión del manuscrito; Adriana Antonio Bautista: análisis estadístico, revisión de la toma de datos, revisión general del manuscrito; Arturo Mancera Rico: revisión del proyecto, correcciones finales, revisión del manuscrito. 


\section{Referencias}

Abdullah, S. K. and K. A. Al-Mosawi 2010. Fungi associated with seeds of sunflower (Helianthus annuus) cultivars grown in Iraq. Phytopathologia. 57:11-20. https://www.cabi.org/ISC/FullTextPDF/2011/20113179658.pdf (20 de junio de 2019).

Arguedas, M. 1997. Plagas de semillas forestales en América Central y el Caribe. CATIE. Turrialba, Costa Rica. 120 p.

Arguedas, M. 2011. Problemas fitosanitarios en teca (Tectona grandis L.f.) en América Central. In: Chavarriaga, D. M. (ed.). Protección fitosanitaria forestal. ICA. Medellín, Colombia. pp: 147-160.

Barnett, H. L. and B. B. Hunter. 1998. Ilustrated genera of imperfect fungi. American Phytopathological Society Press. St. Paul, MN, USA. 218 p.

Borges J., A. y J. Urdaneta. 2010. Efecto de Fusarium sp. en la germinación, fenología y supervivencia de plántulas de Leucaena leucocephala (lam.) de Wit. Agronomía Tropical 60(2): 155-160. http://ve.scielo.org/scielo.php?pid=S0002192X2010000200004\&script=sci_abstract (13 de enero de 2019).

Campo-Aarana, R. O., N. Urango-Esquivel y M. Espitia-Camacho. 2014. Hongos asociados a la semilla de seis forestales nativos, cultivados en el departamento de Córdoba. Fitopatología Colombiana 38(2):27-31.

https://www.researchgate.net/publication/298809860_Hongos_asociados_a_la_semilla_de_seis _forestales_nativos_cultivados_en_el_departamento_de_Cordoba (20 de noviembre de 2018).

Ceballos-Freire, A. J. y J. A. López-Ríos. 2007. Conservación de la calidad de semillas forestales nativas en almacenamiento. Cenicafé 58(4): 265-292. https://www.cenicafe.org/es/publications/arc058\%2804\%29265-292.pdf (24 de enero de 2019). 
Comisión Nacional para el Conocimiento y Uso de la Biodiversidad (Conabio). 2018. Pinus prieto (Pinus greggii) Naturalista. https://www.naturalista.mx/taxa/135782Pinus-greggii (6 de noviembre de 2018).

Comisión Nacional Forestal (Conafor) - Organización de las Naciones Unidas para la Alimentación y la Agricultura (FAO). 2011. Situación de los recursos genéticos forestales en México. http://www.fao.org/3/a-be793s.pdf (15 de octubre de 2018).

Comisión Nacional Forestal (Conafor). 2017a. Paquete tecnológico Pinus greggii Englem. http://www.conafor.gob.mx:8080/documentos/docs/13/961Pinus\%20greggii.pdf (10 de octubre de 2018).

Comisión Nacional Forestal (Conafor). 2017b. Paquete tecnológico Pinus greggii Englem. Pinus montezumae Lamb.

http://www.conafor.gob.mx:8080/documentos/docs/13/971Pinus\%20montezumae. pdf (10 de octubre de 2018).

Christin, Z. L., K. Bagstad and M. Verdone, 2016. A decision framework for identifying models to estimate forest ecosystem services gains from restoration. Forest Ecosystems 3:3. Doi: 10.1186/s40663-016-0062-y.

Delouche, J. C. 1972. Harvesting, handling and storage of soybean seed. In: Proceedings of Short Course for Seedsmen. Mississippi State University. Mississippi, MS, USA. pp. 97-122.

Du Hyun, K. and H. H. Sim. 2018. Seed coat and aging conditions affect germination and physiological changes of aging Korean pine seeds. Journal of Forest Research. 23: 372-379. Doi: 10.1080/13416979.2018.1531478.

Engels, J. y L. Visser. 2007. Guía para el manejo eficaz de un banco de germoplasma. Manuales para Bancos de Germoplasma No. 6. Bioversity International. Roma, Italia. 209p. 
Guerra, C., H. Cruz, I. Vila, A. Duarte y O. M. López. 2004. Principales hongos que afectan a Pinus tropicalis Morelet en Cuba. Fitosanidad 8: 9-12. http://www.fitosanidad.cu/index.php/fitosanidad/article/view/373 (6 de febrero de 2019). Hilli, A., S. Tillman, E. and A. Kauppi, 2003. Germination of pretreated Scots pine seeds after long-term storage. Canadian Journal of Forest Research. 33(1): 47-53. Doi: $10.1139 / \times 02-155$.

International Seed Testing Association (ISTA) 2004. International rules for seed testing. Supplement Rules. Seed Science and Technology. Vol. 24. Zürichstr, Bassersdorf, Switzerland. 335 p.

Lee, S. S. 2011. Diseases of acacias in South-Eas Asia. In: Chavarriaga, D. M. (ed.). Protección fitosanitaria forestal. ICA. Medellín, Colombia. pp. 69-76.

Moreno M., E. 1988. Manual para la identificación de los hongos en granos y sus derivados. Universidad Nacional Autónoma de México. México, D. F. México. 109 p.

Mittal, R. K., R. L. Anderson and S. B. Mathur. 1990. Microorganisms associated with tree seeds. World Checklist. Patawawa National Forestry Institute. Ontario, Canada. 57 p.

Neegaard, P. 1977. Seed pathology. MacMillan Press Ltd. London, UK. 829 p.

Peterson, M. 2008. Fusarium species, a British Columbia perspective in forest seedling production. In: Dumroese, R. K. and L. E. Riley (eds.). Proceedings of the Forest and Conservation Nursery Associations Meetting 2007. Sidney, 17-19 September 2007. USDA Forest Service. Fort Collins, CO, USA pp. 109-125.

Sistema Nacional Inspección y Certificación de Semillas (SNICS). 2018. ¿EI contenido de humedad afecta la calidad de las semillas? Gobierno de México. https://www.gob.mx/snics/articulos/el-contenido-de-humedad-afecta-la-calidad-dela-semilla?idiom=es (20 de septiembre de 2018). 
Skrøppa, T. and K. B. Fjellstad. 2017. Conservation of forest genetic resources in Norway in a climate change perspective. In: Ahuja, M. and S. Jain (eds.).

Biodiversity and conservation of woody plants. Sustainable Development and Biodiversity. 17:129-153. Doi: 10.1007/978-3-319-66426-2_5.

Solano-Bonilla, M. y D. Brenes-Chacón. 2012. Evaluación de métodos de curación de sustratos para la prevención del mal de talluelo. Revista Forestal Mesoamericana. Kurú 9: 63-65. Doi: 10.18845/rfmk.v9i22.365.

Statical Analysis System (SAS). 2002. (Version 9.0). Statical Analysis System Institute Inc. Cary, NC, USA. n/p.

Suma, A., K. Sreenivasan, K. Singh and J. Radhamani. 2013. Role of relative humidity in processing and storage of seeds and assessment of variability in storage behaviour in Brassica spp. and Eruca sativa. The Scientific World Journal Vol. 2013: 1-9. Doi: $10.1155 / 2013 / 504141$.

Wang, B. S. y T. Beardmore. 2004. Almacenamiento y manejo de germoplasma. In: Vargas, H., J. Jesús, B. Bermejo y F. T. Ledig (eds.). Manejo de recursos genéticos forestales. Segunda edición. Colegio de Posgraduados, Montecillo, México y Comisión Nacional Forestal. Zapopan, Jal., México. pp. 107-140.

Winston, P. W. and D. H. Bates. 1960. Saturated solutions for the control of humidity in biological research. Ecology 41: 232-237. Doi: 10.2307/1931961.

\section{(c) (†) (5)}

Todos los textos publicados por la Revista Mexicana de Ciencias Forestales -sin excepciónse distribuyen amparados bajo la licencia Creative Commons 4.0 Atribución-No Comercial (CC BYNC 4.0 Internacional), que permite a terceros utilizar lo publicado siempre que mencionen la autoría del trabajo y a la primera publicación en esta revista. 